\title{
Our experience of laser lithotripsy under local anesthesia in the treatment of bladder stones in obese male patients
}

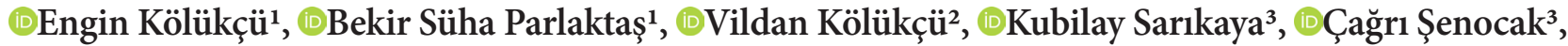 \\ (1) Ömer Faruk Bozkurt ${ }^{3}$ \\ ${ }^{1}$ Tokat Gaziosmanpaşa University, Faculty of Medicine, Department of Urology, Tokat, Turkey \\ ${ }^{2}$ Tokat State Hospital, Department of Anesthesiology and Reanimation, Tokat, Turkey \\ ${ }^{3}$ University of Health Sciences, Keçiören Training and Research Hospital, Department of Urology, Ankara, Turkey
}

Cite this article as: Kölükçü E, Parlaktaş BS, Kölükçü V, Sarıkaya K, Şenocak Ç, Bozkurt ÖF. Our experience of laser lithotripsy under local anesthesia in the treatment of bladder stones in obese male patients. J Health Sci Med 2021; 4(3): 314-321.

\begin{abstract}
Introduction: Our century has witnessed a significant increase in obesity, which in its turn, bears several major risks in management of systemic anesthesia. In this context, use of this treatment without general or regional anesthesia is of critical importance for patients under this group.

Aim: In present study, it was aimed to analyze the efficacy and safety of holmium laser lithotripsy (HLL) under local anesthesia in obese male patients with bladder stones.

Material and Method: In our study, we evaluated a total of 64 obese male patients with body mass index (BMI) $\geq 30 \mathrm{~kg} / \mathrm{m}^{2}$, diagnosed with bladder stones and treated HLL under local anesthesia. Demographic data, stone size, operative time, urethral catheterization time and length of hospital stay of the patients were analyzed. Visual Analogue Scale (VAS) scores was calculated for each patient during the procedure. Complications were graded according to modified Clavien classification system.

Results: The mean age of patients was $50.58 \pm 13.04$ years and BMI was $33.46 \pm 2.59 \mathrm{~kg} / \mathrm{m}^{2}$. Mean stone size was $2.51 \pm 1.04 \mathrm{~cm}$ and operative time was $43.91 \pm 15.92$ minutes. None of the patients had severe pain and the mean VAS score was calculated as $2.31 \pm 1.02$. Mean length of hospital stay was $1.25 \pm 1.04$ days. Grade 3 or higher complications were not observed according to modified Clavien classification system. Mean urethral catheterization time was $1.56 \pm 1.45$ days, although varying due to underlying etiological factor. All patients were stone-free and there was a marked improvement in clinical findings.

Conclusion: Use of HLL under local anesthesia is a safe and efficacious approach in obese male patients with bladder stone.
\end{abstract}

Keywords: Obesity, male, local anesthesia, holmium laser, bladder

\section{INTRODUCTION}

Obesity is a considerably complex and multifactorial pathology. Body mass index (BMI) is widely used to define obesity all around the world. Individuals with BMI of 30 and over are considered as obese by the World Health Organization (WHO). This century has witnessed ahighlevel of increase in prevalence of obesity in all parts of the world. This applies not only to countries with high-income level but also those with middle and low-income level. While prevalence of obesity was reported as $3.2 \%$ in males and $6.4 \%$ in females in 1975 in large-scale epidemiological studies, it was reported to increase to a high level of $10.8 \%$ in males and $14.9 \%$ in females in 2014 (1). Obesity is at the moment considered as a world-wide epidemic by several authors. Recent clinical studies have concluded that $57.8 \%$ of adults may be diagnosed with obesity until 2030 (2). In parallel to the increase in prevalence of obesity, it is obvious that the health professionals would be more intensively dealing with this patient group in the near future. All systems in body can be negatively affected due to obesity. Recent studies have revealed that obesity is closely associated with many clinical conditions such as hypertension, dyslipidemia, diabetes mellitus, cardiovascular diseases, musculoskeletal disorders, sleep-apnea syndrome and liver diseases. In addition to this, anesthesiologists also experience major challenges in airway management of obese patients as a result of enlargement in the face, neck, chest and abdomen area. These factors put obese patients at a very high-risk group in terms of general or regional anesthesia. (3). For this reason, interventions under local anesthesia are of critical importance for obese patients. 
Urinary stone disease is a very common pathology in practice of urology. Large-scale epidemiological studies conducted in the past reported prevalence rates as ranging between $4 \%$ and $20 \%$ (4). Besides, bladder stones represent only $5 \%$ of urinary stone disease. Clinical manifestations are differing from the upper urinary tract stones, which are detected more commonly. Most common symptoms are acute urinary retention, dysuria, pollakiuria, hematuria, weak urine flow and suprapubic pain. Many different protocols such as cystolithotomy, transurethral cystolithotripsy, extra corporeal shock wave lithotripsy (ESWL) and percutaneous suprapubic cystolitholapaxy have been used in the treatment of bladder stones. Size and composition of the stone, clinical status of the patient and the underlying etiological factors causing stone formation play a critical role in identifying the treatment strategies (5). Nevertheless, our knowledge about treatment algorithms is relatively less when compared to upper urinary tract stones. Besides, majority of the studies on treatment of bladder stones performed in the past years mainly analyzed surgical interventions under general or regional anesthesia. Our study is the first in literature for analyzing the efficacy and safety of holmium laser lithotripsy (HLL) under local anesthesia for treatment of bladder stones in obese male patients.

\section{MATERIAL AND METHOD}

This retrospective study was carried out following the principles of the Helsinki Declaration and with the approval of theGaziosmanpaşa University Clinical Researchs Ethics Committee (Date: 03.12.2020, Decision No: 20-KAEK-295).

Retrospective clinical data of 64 male patients with BMI $\geq 30 \mathrm{~kg} / \mathrm{m}^{2}$ who underwent HLL under local anesthesia for bladder stones in our clinics between January 2018-October 2020 were evaluated. Detailed medical history of the patients was taken, physical examinations were completed and their demographics were recorded. Charlson Comorbidity Index was used to analyze clinical conditions of the patients (6). In addition to this, preoperative lower urinary tract complaints were scored by using International Prostate Symptom Score (IPSS) and Quality of Life (QoL) (7). Uroflowmetry test and post-void residual volume were used to identify voiding patterns. Diagnostic steps, assessing lower urinary tract symptoms of patients with acute urinary retention, were not taken. Complete urinalysis, urine culture and routine biochemistry and hematology tests were done for all patients prior to endoscopic interventions. Radiological investigations included kidney, ureter, bladder (KUB) x-ray, urinary ultrasonography and non-contrast tomography.
Suprapubic cystostomy catheter was firstly placed in 27 patients with acute urinary retention under emergency conditions. Supine position was preferred for endourological approach in cases with severe posture deformity, such as hip and spinal disorders, which do not allow for the lithotomy position. Rest of the patients were operated routinely under lithotomy position. Antibiotic treatment was given to patients with positive urine culture. The patients were operated after confirmation of negative urine culture. The heart rate, arterial pressure, ventilation frequency and peripheral oxygen saturation were recorded during the surgery. It was planned to terminate the procedure in case of any deterioration in vital signs, any major complication or high level of pain.

All endourological procedures were performed under sterile conditions by using flexible cystoscope. A single dose $1000 \mathrm{mg}$ of the first-generation cephalosporin was administered routinely to all patients 1 hour before the procedure. Pethidine HCI (Aldolan vial $100 \mathrm{mg} / 2$ $\mathrm{mL}$, Vem, Turkey) was administered to the patients as a premedication at a dose of $50 \mathrm{mg}$ intramuscularly. Before the endourological intervention, $10-\mathrm{cc}$ of $2 \%$ lidocaine gel was instilled and a penile clamp was placed to make sure that the gel stayed at the urethra. After a waiting time of 15 minutes, same amount of lidocaine gel was applied into the urethra just before the flexible cystoscope was inserted to the anterior urethra. When a urethral stricture was detected during cystourethroscopy (Karl Storz, United States), an s-curve dilatation was performed with a guidewire. In addition, it was decided not to perform the procedure under local anesthesia and also to include prostate surgery as a part of the procedure, in case prostate lobes were found to be large and closing the urethra as a result of endourological evaluation. Such group of patients were not included in the study.

Holmium: yttrium-aluminum-garnet laser device was used as the lithotripter. During lithotripsy, two different probes, $272 \mu$ and $365 \mu$, were used depending on the size of the stone. The laser energy was set at 0.5-1 Joule J and 5-40 Hz. Sterile $0.9 \%-\mathrm{NaCl}$ solution was used as irrigation fluid during the procedure. After the procedure, the patients were followed-up with three-way $18 \mathrm{~F}$ catheter and irrigation fluid for a period of 6-8 hours to facilitate drainage of stone fragments. Pain level of the patients was analyzed immediately after the surgery by using visual analogue scale (8). Furthermore, modified Clavien classification system was used to grade intra and postoperative complications (9). In addition to this, length of hospital stay, need for treatment during follow-up at hospital and timing of urethral catheter removal were evaluated. The patients were asked to visit the hospital for follow-up in the first postoperative month. Uroflowmetry test, IPSS, QoL and urinary ultrasonography results were recorded. 
Only obese male patients with bladder stones were included in our study. The patients signed written informed consent form after detailed briefing on the modalities to be applied for treating bladder stones and on local anesthesia, prior to the start of procedure. Cases with acute urinary tract infections, any history of allergy to local anesthetics or those having any neurological pathology, severe mental or psychological disorders were excluded from the study. Endourological procedures under local anesthesia were not applied to patients requiring any surgery for bladder cancer at the same time or patients having a prostatic hyperplasia at a size covering the entire urethra.

\section{Statistical Method}

Descriptive analyses were performed in order to give information on the general characteristics of the groups. Data was given as Mean \pm Standard Deviation. PairedSample T test was used to analyze the difference between the groups in repeated measures. Pearson's Correlation Coefficient was used to measure the association between quantitative data. $\mathrm{P}$ value less than 0.05 is considered as statistically significant. Commercially available statistical software was used for calculation purposes. (IBM SPSS Statistics 19, SPSS inc., an IBM Co., Somers, NY).

\section{RESULTS}

Patients demographics and treatment characteristics are shown in Table 1. Mean age of the patients was $50.58 \pm 13.04$ years and BMI was calculated as $33.46 \pm 2.59$ $\mathrm{kg} / \mathrm{m}^{2}$. The main findings were acute urinary retention in $27(42.2 \%)$ patients, intermittent hematuria in $16(25 \%)$, weak urine stream in $12(18.7 \%)$, dysuria in $5(7.8 \%)$, frequency in $3(4.7 \%)$ and urethrorrhagia in one $(1.6 \%)$ patient. Urine culture was positive in 28 (43.8\%) patients. Most common pathogen was Escherichia coli (53.6\%). All patients had minimum one chronic disease. Most common pathology was peptic ulcer and hyperlipidemia. Charlson comorbidity index was calculated as $4.55 \pm 2.84$. The smallest stone size was $0.9 \mathrm{~cm}$ and the largest stone size was $4.5 \mathrm{~cm}$. The mean stone size was $2.51 \pm 1.04 \mathrm{~cm}$. More than one bladder stone was detected in a total of 7 (10.9\%) patients.

\begin{tabular}{|lcc|}
\hline Table 1. Patients demographics and treatment characteristics \\
\hline Mean & $\begin{array}{c}\text { Standard } \\
\text { deviation }\end{array}$ \\
\hline Age (year) & 50.58 & 13.04 \\
Body mass index $\left(\mathrm{kg} / \mathrm{m}^{2}\right)$ & 33.46 & 2.59 \\
Charlson comorbidity index & 4.55 & 2.84 \\
Stone size $(\mathrm{cm})$ & 2.51 & 1.04 \\
Operative time (minute) & 43.91 & 15.92 \\
VAS score & 2.31 & 1.02 \\
Urethral catheterization time (days) & 1.56 & 1.45 \\
Length of hospital stay (days) & 1.25 & 1.04 \\
\hline VAS: Visual analogue scale & & \\
\hline
\end{tabular}

A total of $30(46.9 \%)$ patients were diagnosed with benign prostatic hyperplasia, which is interpreted as the main most common predisposing factor for bladder stone formation. When the other etiological factors are concerned, $10(15.6 \%)$ patients had urethral stricture and a foreign body was detected in the bladder of one case (1.6\%). No anatomical anomaly that may result in bladder stone formation was found in $23(35.9 \%)$ patients. A total of 35 (54.7\%) patients had history of urinary stone disease. Although it was decided not to include the patients having a prostate tissue at a size covering the entire urethra in the study, the above mentioned endourological procedures were applied to cases with mild to moderate level of prostatic hyperplasia after starting early alpha-blocker therapy.

Mean operative time was $43.91 \pm 15.92$ minutes and urethral dilatation was done in $10(15.6 \%)$ patients. All bladder stones were successfully fragmented and all patients were stone-free. All surgical interventions were performed at lithotomy position, except for 3 (4.7\%) cases with severe posture deformities. Any condition such as massive hematuria or bladder perforation that may require termination of endourological stone surgery, was not experienced during the procedures. The mean VAS was calculated as $2.31 \pm 1.02$. There was no patient with severe pain during the surgery and no patient required analgesics after the procedure. We found a high level, positive and significant relationship between the VAS and stone size $(\mathrm{r}=0,741 ; \mathrm{p}<0,001)$. Again, there was a weak, positive and significant relationship between the VAS and operative time $(\mathrm{r}=0,397 ; \mathrm{p}=0,001)$. However, there was no significant relationship between VAS and age and BMI ( $\mathrm{p}=0.952$ and $\mathrm{p}=0.336$, respectively) (Table 2$)$.

\begin{tabular}{|lccccc|}
\hline \multicolumn{5}{|l|}{ Table 2. Correlation between Visual Analogue Scale scores and } \\
stone size, operative time, age and Body Mass Index \\
\hline \multicolumn{7}{|c|}{$\begin{array}{c}\text { Stone size } \\
\text { (cm) }\end{array}$} & $\begin{array}{c}\text { Operative time } \\
\text { (Minute) }\end{array}$ & $\begin{array}{c}\text { Age } \\
\text { (year) }\end{array}$ & $\begin{array}{c}\text { BMI } \\
\left(\mathbf{k g} / \mathbf{m}^{2}\right)\end{array}$ \\
\hline $\begin{array}{l}\text { VAS } \\
\text { score }\end{array}$ & $\mathrm{r}$ & 0.741 & 0.397 & 0.008 & 0.122 \\
\hline & $\mathrm{p}$ & $<0.001^{\star}$ & $0.001^{\star}$ & 0.952 & 0.336 \\
\hline
\end{tabular}

BMI: Body Mass Index; VAS: Visual Analog Scale; r: Pearson's correlation coefficient. $\mathrm{p}$ value is significant at the level of 0.05

Patients without urethral stricture were followed-up for one day under urethral catheterization. In patients with urethral stricture, duration of catheter ranged from 3 to 5 days. Mean catheterization time was $1.56 \pm 1.45$ days. None of the patients developed acute urinary retention after removal of urethral catheter. Minor complications developed in a total of $13(20.3 \%)$ patients after the procedure. A total of $8(12.5 \%)$ patients developed hematuria, which lasted less than 24 hours and did not require blood transfusion and $2(3.1 \%)$ patients had urethrorrhagia, which recovered without any additional intervention. A total of $3(4.7 \%)$ patients had 
postoperative fever. When the fever etiology of these patients was analyzed in detail, it was found to develop secondary to urinary tract infection. None of the patients required intravenous antibiotic treatment and fever was taken under control in a short time with medical therapy. None of the patients had any complication that require hospitalization. When postoperative complications were graded according to the modified Clavien classification, $10(15.6 \%)$ patients had Grade 1 and 3 (4.7\%) patients had Grade 2 complications. However, no patient had any complication of Grade 3 and above that would cause mortality or morbidity according to the modified Clavien classification.

After exclusion criteria were met, mean preoperative IPSS and Qol values were found to be $19.35 \pm 7.78$ and $4.03 \pm 1.30$, respectively. Similarly, maximum flow rate was measured as $9.19 \pm 3.49 \mathrm{~mL} / \mathrm{s}$ in the uroflowmetry test. Mean post-void residual volume was calculated as $58.51 \pm 20.91 \mathrm{cc}$ at the urinary ultrasonography. Mean IPSS and Qol values were found to be $9.08 \pm 2.15$ and $1.86 \pm 0.71$, respectively, in the first month follow-up postoperatively. Maximum urinary flow rate was calculated as $17.64 \pm 3.95$ $\mathrm{mL} / \mathrm{s}$. Mean post-void residual volume was $23.51 \pm 11.54$ cc. A statistically significant improvement was observed in the prostate symptom scores, uroflowmetry test results and ultrasonography findings after the endourological intervention $(\mathrm{p}<0.001)$.

\section{DISCUSSION}

Bladder stones are pathologies known since times before Christ. In endemic regions, bladder stones are observed in childhood in parallel with eating habits, but in western societies, they are mostly diagnosed at the age of $60 \mathrm{~s}$. Prostatic hyperplasia is amongst the most common etiological factors. Nevertheless, bladder stones may be secondary to many clinical conditions including urethral strictures, bladder neck contractures, foreign bodies and neurogenic bladder. Douenias et al. (10) presented a series of 100 patients reporting up to $88 \%$ of the cases bladder outlet obstruction. In a similar study, Tzortzis et al. (11) reported prostatic hyperplasia in $51.6 \%$ of the cases diagnosed with bladder stone. In our study, age average of the patients is $50.58 \pm 13.04$ years with the most common etiological factor being prostate hyperplasia (46.9\%). Low urinary $\mathrm{pH}$, decreased magnesium level and increased urinary uric acid supernaturation secondary to urinary stasis are quite critical in the formation of bladder stones. Previous analyses report history of urinary stone disease in more than $1 / 3$ of the bladder stone cases (12). Our study indicates urinary stone disease diagnosed in the history of $54.7 \%$ of our cases. In addition, there is a close association between obesity and urolithiasis. Previous clinical trials report that up to $98 \%$ of obese patients present at least one of the lithogenic risk factors such as hypercalciuria, hyperoxaluria, low urinary volume or hyperinsulinemia (13). We expect a critical increase in the number of obese patients with bladder stones applying to clinics as a result of the aging world population. On the other hand, we are in the opinion that anesthesiology and the surgical technique to be followed have a grave impact on minimizing mortality and morbidity in this group of patients with many comorbidities.

Both general and regional anesthesia to be applied in obese patients come with high mortality and morbidity in obese patients in many aspects. The first challenge in obese patients is the anatomical factors. The neck is rather short, larynx changes position to the anterosuperior and the tongue is overgrown in the obese. Altogether, these make preanesthetic intubation a big challenge. In their series of 263 cases, Juvin et al. (14) reported a difficult intubation ratio up to $15.5 \%$ in obese patients, which is at the level of $2.2 \%$ in patients with a BMI under 30 . Thoracic compliance diminishes in obese patients since the diaphragm changes position superiorly and its movements are challenged. These anatomical changes pose a negative effect on the lung functions increasing the risk of intra and postoperative atelectasis in obese patients (3). In a series of 407 bariatric surgery patients, Baltieri et al. (15) reported atelectasis in more than one third of their cases. Another quite common phenomenon is the sleep apnea syndrome in obese patients. Previous studies report a considerable level of sleep apnea syndrome in almost $20 \%$ of obese patients. This respiratory pathology is closely associated with postoperative desaturation, respiratory failure, and some cardiac problems including atrial fibrillation (16). Increases in BMI are accepted as serious risk factors for cardiovascular diseases. A large scale trial conducted in Europe reports a 37\% prevalence of cardiovascular diseases in cases with a BMI over 30 (17). Cardiac output, circulating blood volume and sympathetic activity tend to increase in the obese population, which is directly associated with the incidence of hypertension, cardiomegaly, congestive heart failure, coronary artery disease, peripheral vascular disease and thromboembolism $(3,16)$. These all contribute to anesthesia-related intra and postoperative complication risks. Obese patients also present with diminished lower esophageal sphincter pressure. Once the intraabdominal pressure is increased, gastroesophageal reflux gets quite common in this patient population. This condition puts obese patients in a risky position for aspiration before induction of anesthesia. The most common problems observed in the obese population in relation to regional anesthesia are increased skin epidural distance, diminished cerebrospinal fluid volume and narrow epidural space. These clinical conditions lead to unfavorable results in regional anesthesia and 
lower its success rates (3). Considering all these factors together, any therapeutic intervention without general or regional anesthesia is quite critical for obese patients. We are in the opinion that our study would provide insight to treatment of obese bladder stone cases under local anesthesia for the first time ever in the literature.

Today, health professionals prefer minimally invasive modalities for treatment of bladder stones. On the other hand, clinical data on management of bladder stones are quite limited in the literature. In a review by Torricelli et al., the authors discussed success rates of methods applied in the treatment of bladder stones. They reported stone-free rates of $100 \%$ in open surgery, $75-100 \%$ in ESWL, $89-100 \%$ in percutaneous cystolithotripsy and $63-100 \%$ in transurethral cystolithotripsy (18). In another more recent study, success rates of endoscopic and open surgical techniques were reported as 92.5\% and $100 \%$, respectively. Again in the same study, authors concluded that open surgery presents higher complication rates than the endoscopic approach (19). Currently, it is well known that open surgery is the technique of choice for limited indications such as cases with oversized bladder stones or those, who are scheduled to undergo simultaneous prostatectomy or diverticulotomy (20). In obese patients, on the other hand, open surgical approach should be the last resort in our opinion. Open surgery poses two levels of problems for this group of patients. One problem is that open surgery contains procedures that would be unsafe for the patient without general or regional anesthesia, and the second is that wound healing is impaired in obese patients.

Extra corporeal shock wave lithotripsy is a significant minimally invasive treatment option for urinary stone disease. Nevertheless, its use in bladder stones is not as prevalent as in upper urinary system stones. There are two main reasons for that: i. lack of evidence in etiological factors of patients undergoing only ESWL; ii. insufficiency of ESWL devices in achieving required level of fragmentation particularly in oversized stones resulting in difficulty in evacuation of fragments . Recent analyses highlight the need for endourological procedures in up to $17 \%$ of post-ESWL bladder stone cases for clearance of stone fragments (5). Another minimally invasive treatment option that can be applied in treatment of bladder stones is the percutaneous procedures. Percutaneous suprapubic approach has been used for more than a decade in treatment of bladder stones. This technique can be applied under either general or regional anesthesia although there are also centers applying the technique under local anesthesia. The first successful series of bladder stones was by Tzortzis et al. (11) in the literature. They reported having applied percutaneous cystolithotripsy to 31 bladder stone patients under local anesthesia and that all patients tolerated this approach quite well. The same study also reported a stone-free rate of up to $96.78 \%$, and no patient developed major intraoperative complications. A similar study by Nashar et al. (21) presented $95 \%$ success rate in a series of 42 cases having undergone percutaneous cystolithotripsy under local anesthesia. They also indicated that severe hematuria developed in one patient and that they had to perform a second surgical operation under deep analgesia for management of this situation. The biggest advantage of this method is that it makes it possible for oversized stones to be fragmented and evacuated within a very short time without causing urethral damage. On the other hand, the need for a second urethral intervention such as transurethral prostate resection or internal urethrotomy can be listed among its disadvantages. Percutaneous cystolithotripsy was not preferred in our series of obese male patients with bladder stones. Due to presence of an intensive adipose tissue in obese patients, suprapubic access under local anesthesia can be quite challenging in our opinion. So, we have the concern that patient's pain level may not be effectively controlled and surgical complication rates may raise as a consequence. Due to all these reasons, this minimally invasive approach was not used primarily in our cases.

Today, surgical procedures that are conducted through the natural orifices of the body have gained importance with their early healing times and short hospital stays. Urologists prefer to follow this approach in many treatment modalities including transurethral prostate resection, retrograde intrarenal surgery, endoscopic cystolithotomy and internal urethrotomy. So, transurethral cystolithotripsy is quite extensively used in the treatment of bladder stones. Although various types of energy sources such as electrohydraulic, ultrasonic ve pneumatic powered instruments were used commonly in the past, laser technology has now started a new era in the treatment of stone diseases. With this stateof-the-art power being introduced into medicine and with increasing surgical experience, complications of transurethral cystolithotripsy such as hematuria, bladder perforations, urinary system infections and mucosal injuries have decreased dramatically (22). Holmium: holmium-yttrium-aluminum-garnet laser is the most commonly and effectively used type of lithotripsy with a wavelength of $2120 \mathrm{~nm}$ and maximum absorbability in water feature. The system provides regular, symmetrical and small stone fragmentation by use of mainly photothermal mechanism. This is, in particular, a major advantage in natural evacuation of stones (23). Considering our literature knowledge so far, it is observed that holmium laser can be used safely and successfully in the treatment of bladder stones. 
In a study by Teichman et al. (24), holmium laser cystolithotripsy was performed on bladder stones bigger than $4 \mathrm{~cm}$ under general or spinal anesthesia and all the patients were defined stone free with $85.7 \%$ of the cases discharged on the first postoperative day. The study by Shah et al. (25) examined 32 male patients diagnosed with bladder stones in association to bladder outlet obstruction. After having applied holmium laser enucleation of the prostate simultaneously with transurethral holmium laser cystolithotripsy, they reported their success rate as $100 \%$. The same study also reported intra and postoperative complication rates of $12.5 \%$ and $15.6 \%$, respectively declaring no major complication in any of the patients. The literature presents limited number of studies discussing efficacy of transurethral cystolithotripsy in treatment of bladder stones under local anesthesia. In a series of 46 patients, Garg et al. (26) reported a success rate of $97.6 \%$ for transurethral treatment of bladder stones by use of holmium laser under local anesthesia explaining that $95.7 \%$ of the patients could well tolerate the procedure. Following a similar approach, Nameirakpam et al. (27) assessed 85 cases with bladder stones of $1.5 \mathrm{~cm}$ and above reporting that all patients got stone free without the need for hospitalization. The same study also reported that $5.9 \%$ of the patients had severely elevated VAS. We are in the opinion that this factor is directly linked to the use of 15/19 f semi rigid cystoscope. We have used, in our study a relatively new technology, the flexible cystoscopes and observed no severe VAS elevation in any of our patients. Patients with urethral strictures were not included in either of the clinical analyses. We have successfully dilated urethral strictures of 10 cases $(15.6 \%)$. In both of these studies, stone fragments were evacuated by use of an aspirator or evacuator. In our study, on the other hand, the fragments were so much fragmented that we could allow for evacuation by natural micturition. As regards operative time, none of the cases exceeded 1.5 hours. We are in the opinion that this is associated with our clinical experience and high level technical conditions that were available to us. The results of using transurethral holmium laser technology concomitantly with flexible cystoscopy under local anesthesia were first published by Kara et al. (22) in a series of 13 male patients. Thy reported a 100\% success rate and explained that no patient needed any extra analgesic. On the other hand, they reported only 1 case developing fever in reaction to conservative therapy. Similarly, we also had 3 patients developing fever after medical therapy but their fever regressed to medical therapy. Although the procedures are performed under fully sterile conditions, unfavorable effects of obesity on the immune system and composition of the stones may be responsible for this situation. In their series of
37 male patients with bladder stones in an average size of $2.1 \mathrm{~cm}$, D'Souza and Verma (20) performed holmium laser cystolithotripsy by flexible cystoscope under local anesthesia. They reported complete stone clearance and no major complication. Yet, they reported that $18.9 \%$ of the cases had to undergo recatheterization due to prostate hyperplasia on the first day after removing the catheter. None of the cases needed recatheterization in our study. This can be explained in two ways: one is that none of the patients, who needed prostate surgery underwent bladder stone intervention under local anesthesia during endoscopic assessment; and the other that alpha blockers were started early in cases mild to moderate level of prostatic hyperplasia. Another transurethral approach in treatment of bladder stones with local anesthesia is the use of semirigid ureteroscopes. For the first time in the literature, Uzun et al. (28) discussed the results of this technique in a series of 18 patients and reported a success rate of up to $89 \%$. The same study also reported a procedure time of 30 minutes and more in only $12.5 \%$ of the cases. Considering the physical condition of obese patients, we have not preferred semirigid uretheroscopes in our study due to concerns that this technique may not provide a smooth surgery in fragmentation of bladder stones.

Today, there are various clinical approaches that can be applied in treatment strategies of prostatic hyperplasia cases with bladder stone. In a study by O'Connor et al. (29), 23 cases developing bladder stones secondary to prostate hyperplasia were discussed. In their studies, endourological treatment methods were preferred for bladder stones, while medical treatment was applied for prostatic hyperplasia. They detected a statistically significant level of improvement in the symptom scores and postvoid residual urine volume values of all patients postoperatively. Furthermore, recurrent calculi were reported in only $17.4 \%$ of the patients. Philippou et al. (30) analyzed the efficacy of medical treatment and transurethral prostate resection in 64 patients with bladder stones. They documented in their study that the group having undergone prostate surgery presented with a more significant improvement in terms of symptom scores and uroflowmetry values, and yet unsuccessful results were reported in $34.3 \%$ of the cases having received only medical treatment. In our study, we spared prostate surgery only for cases with large prostate lobes covering the urethra. This was mainly due to the fact that our cases presented high anesthesiological risks due to their comorbidities. Alpha blocker treatment was started early for every case diagnosed with prostate hyperplasia. Yet, it was considered a better approach to refer these patients to a nutrition specialist for better weight control if they are to be scheduled for prostate hyperplasia surgery. 
The limitations of our study were mainly about sample size and short follow-up time. It is another limitation that the patients could not be subject to detailed metabolic work-up due to technical unavailabilities and that the stone analyzes could not be documented.

\section{CONCLUSION}

To our knowledge, this is the first study examining the use of the holmium laser for the treatment of bladder stones in obese male patients. The data obtained in our study indicated the use of HLL has effective results in the obese male patients with low complication rates. We think that our data should be supported by randomized, largescale and prospective studies in which comprehensive biochemical findings are presented and include long follow-up periods.

\section{ETHICAL DECLARATIONS}

Ethics Committee Approval: This retrospective study was carried out following the principles of the Helsinki Declaration and with the approval of the Gaziosmanpaşa University Clinical Researchs Ethics Committee (Date: 03.12.2020, Decision No: 20-KAEK-295).

Informed Consent: Because the study was designed retrospectively, no written informed consent form was obtained from patients.

Referee Evaluation Process: Externally peer-reviewed.

Conflict of Interest Statement: The authors have no conflicts of interest to declare.

Financial Disclosure: The authors declared that this study has received no financial support.

Author Contributions: All of the authors declare that they have all participated in the design, execution, and analysis of the paper, and that they have approved the final version.

\section{REFERENCES}

1. Yosuke I, Bo Q, Jennifer P, Rebeccah S, Penny GL. Epidemiology of obesity in adults: latest trends. Curr Obes Rep 2018; 7: 276-88.

2. Yu S, Xing L, Du Z, et al. Prevalence of obesity and associated risk factors and cardiometabolic comorbidities in rural northeast China. Biomed Res Int 2019; 2019: 6509083.

3. Uludağ Ö, Türktan M. Anesthetic management of patients with obesity. Arch Med Rev J 2016; 25: 406-19.

4. Trinchieri A. Epidemiology of urolithiasis. Arch Ital Urol Androl 1996; 68: 203-49.

5. Cicione A, DE Nunzio C, Manno S, et al. Bladder stone management: an update. Minerva Urol Nefrol 2018; 70: 53-65.

6. Charlson M. Wells MT, Ullman R, King F, Shmukler C. The Charlson comorbidity index can be used prospectively to identify patients who will incur high future costs. PLoS ONE 2014; 3 e112479.
7. Cam K, Müezzinoğlu T, Kayıkçı A, Aydemir Ö. Validity and reliability of Turkish version of quality of life scale specific for partners of patients with benign prostatic hyperplasia. Turkiye Klinikleri J Nephrol 2010; 5: 1-5.

8. Downie WW, Leatham PA, Rhind VW, Wright V, Branco JA, Anderson JA. Studies with pain rating scales. Ann Rheum Dis 1978; 37: 378-81.

9. Dindo D, Demartines N, Clavien PA. Classification of surgical complications: A new proposal with evaluation in a cohort of 6336 patients and results of a survey. AnnSurg 2004: 240: 20513.

10.Douenias R, Rich M, Badlani G, Mazor D, Smith A. Predisposing factors in bladder calculi Review of 100 cases. Urology 1991; 37: 240-3.

11.Tzortzis V, Aravantinos E, Karatzas A, Mitsogiannis IC, Moutzouris G, Melekos MD. Percutaneous suprapubic cystolithotripsy under local anesthesia. Urology 2006; 68: 38-41.

12. Childs MA, Mynderse LA, Rangel LJ, Wilson TM, Lingeman JE, Krambeck AE. Pathogenesis of bladder calculi in the presence of urinary stasis. J Urol 2013; 189: 1347-51.

13. Aydoğdu O. Urinary stone disease and obesity: different pathologies sharing common biochemical mechanisms. World J Nephrol 2012; 1: 12-5.

14.Juvin P, Lavaut E, Dupont H, et al. Difficult tracheal intubation is more common in obese than in lean patients. Anesth Analg 2003; 97: 595-600.

15.Baltieri L, Peixoto-Souza FS, Rasera-Junior I, Montebelo MI, Costa D, Pazzianotto-Forti EM. Analysis of the prevalence of atelectasis in patients undergoing bariatric surgery. Rev Braz J Anesthesiol 2016; 66: 577-82.

16. Pouwels S, Buise MP, Twardowski P, Stepaniak PS, Proczko M. Obesity surgery and anesthesiology risks: a review of key concepts and related physiology. Obes Surg 2019; 29: 2670-7.

17. Adam JP, Murphy PG. Obesity in anaesthesia and intensive care. Br J Anaesth 2000; 85: 91-108.

18. Torricelli FCM, Mazzucchi E, Danilovic A, Coelho RF, Srougi M. Surgical management of bladder stones: literature review. Rev Col Bras Cir 2012; 40: 227-33.

19.Demirdağ Ç, Özman O, Çitgez S, Selçuk B, Önal B, Talat Z. Are the complication rates for the open approach in the surgical treatment of bladder stones higher than rates for endoscopic approaches? a comparative multivariate analysis study. J Uro Surg 2018; 5: 165-9.

20.D'Souza, Verma A. Holmium laser cystolithotripsy under local anaesthesia: Our experience. Arab J Urol 2016; 14: 203-6.

21.Papatsoris AG, Varkarakis I, Dellis A, Deliveliotis C. Bladder lithiasis: from open surgery to lithotripsy. Urol Res 2006; 34: 163-7.

22.Kara C, Resorlu B, Cicekbilek I, Unsal A. Transurethral cystolithotripsy with holmium laser under local anesthesia in selected patients. Urology 2009; 74: 1000-3.

23.Gücük A, Kemahlı E. Urolithiasis and Laser. Turk Urol Sem 2011; 2: 142-5.

24.Teichman JM, Rogenes VJ, McIver BJ, Harris JM. Holmium: yttrium-aluminum-garnet laser cystolithotripsy of large bladder calculi. Urology 1997; 50: 44-8.

25.Shah HN, Hegde SS, Shah JN, Mahajan AP, Bansal MB. Simultaneous transurethral cystolithotripsy with holmium laser enucleation of the prostate: a prospective feasibility study and review of literature. BJU Int 2007; 99: 595-600.

26.Garg M, Kumar M, Goel A, Sankhwar SN. Application of the holmium laser lithotripsy for bladder stones under local anaesthesia: a prospective analysis. Urologia 2015; 82: 219-22. 
27. Nameirakpam S, Naorem S, Faridi MS, Akoijam KS, Sinam RS. Transurethral cystolithotripsy of large bladder stones by Holmium laser as a day care procedure. J Clin Diagn Res 2016; 10: 1-2.

28. Uzun H, Tomak Y, Zorba OU, Bostan H, Kalkan M. Transurethral cystolithotripsy with a ureteroscope under local urethral anaesthesia and sedoanalgesia. J Pak Med Assoc 2013; 63: 961-4.

29. O'Connor RC, Laven BA, Bales GT, Gerber GS. Nonsurgical management of benign prostatic hyperplasia in men with bladder calculi. Urology 2002; 60: 288-91.

30. Philippou P, Volanis D, Kariotis I, Serafetinidis E, Delakas D. Prospective comparative study of endoscopic management of bladder lithiasis: is prostate surgery a necessary adjunct? Urology 2011; 78: 43-7. 